\title{
X-ray Spectra and Light Curves of Cooling Novae and a Nova-Like
}

\author{
Bangzheng Sun, ${ }^{1 \star}$ Marina Orio, ${ }^{1,2} \dagger$ Andrej Dobrotka, ${ }^{3}$ Gerardo Juan Manuel Luna, $, 4,5,6$ \\ Sergey Shugarov ${ }^{7,8}$ and Polina Zemko 9 \\ ${ }^{1}$ Department of Astronomy, University of Wisconsin-Madison, Madison, WI 53715, U.S.A. \\ ${ }^{2}$ INAF - Padova Observatory, vicolo Osservatorio, 5 - 35122 Padova, Italy \\ ${ }^{3}$ Advanced Technologies Research Institute, Faculty of Materials Science and Technology in Trnava, Slovak University of Technology in Bratislava, \\ Bottova 25, 917 24, Trnava, Slovakia \\ ${ }^{4}$ CONICET - ACONICET-Universidad de Buenos Aires, Instituto de Astronomía y Física del Espacio (IAFE), Av. Inte. Güiraldes 2620, C1428Z1 \\ Buenos Aires, Argentina \\ ${ }^{5}$ Universidad de Buenos Aires, Facultad de Ciencias Exactas y Naturales, Buenos Aires, Argentina \\ ${ }^{6}$ Universidad Nacional de Hurlingham, Av. Gdor. Vergara 2222, Villa Tesei, Buenos Aires, Argentina \\ ${ }^{7}$ Astronomical Institute of the Slovak Academy of Sciences, 05960 Tatransk'a Lomnica, The Slovak Republic \\ ${ }^{8}$ P.K. Sternberg Astronomical Institute, M.V. Lomonosov Moscow State University, Russia \\ ${ }^{9}$ University of Padova, 35122 Padova, Italy
}

Accepted XXX. Received YYY; in original form ZZZ

\begin{abstract}
We present X-ray observations of novae V2491 Cyg and KT Eri about 9 years post-outburst, of the dwarf nova and post-nova candidate EY Cyg, and of a VY Scl variable. The first three objects were observed with XMM-Newton, KT Eri also with the Chandra ACIS-S camera, V794 Aql with the Chandra ACIS-S camera and High Energy Transmission Gratings. The two recent novae, similar in outburst amplitude and light curve, appear very different at quiescence. Assuming half of the gravitational energy is irradiated in X-rays, V2491 Cyg is accreting at $\dot{m}=1.4 \times 10^{-9}-10^{-8} M_{\odot} / y r$, while for KT Eri, $\dot{m}<2 \times 10^{-10} M_{\odot} / y r$. V2491 Cyg shows signatures of a magnetized WD, specifically of an intermediate polar. A periodicity of $\sim 39$ minutes, detected in outburst, was still measured and is likely due to WD rotation. EY Cyg is accreting at $\dot{m} \sim 1.8 \times 10^{-11} M_{\odot} / y r$, one magnitude lower than KT Eri, consistently with its U Gem outburst behavior and its quiescent UV flux. The X-rays are modulated with the orbital period, despite the system's low inclination, probably due to the X-ray flux of the secondary. A period of $\sim 81$ minutes is also detected, suggesting that it may also be an intermediate polar. V794 Aql had low X-ray luminosity during an optically high state, about the same level as in a recent optically low state. Thus, we find no clear correlation between optical and X-ray luminosity: the accretion rate seems unstable and variable. The very hard X-ray spectrum indicates a massive WD.
\end{abstract}

Key words: X-rays: stars, stars: cataclysmic variables, novae: individual: V2491 Cyg, KT Eri, EY Cyg, V794 Aql

\section{INTRODUCTION}

We examined quiescence X-ray observations of V2491 Cyg and KT Eri, two of the most luminous novae of the last 12 years, and compared them with EY Cyg, a dwarf nova that is also an old nova candidate, likely to have had an outburst hundreds of years ago, and with the VY Scl system V794 Aql, a nova-like that is thought to be accreting at a high rate, yet does not seem to ever undergo nova eruptions. We note that the possibility that VY Scl systems do have nova outbursts at some point has recently become very interesting after one of them has been observed in a nova outburst (Li et al. 2020).

Preliminary results of this work were published in Sun et al. (2020). The theoretical models of the evolutionary cy-

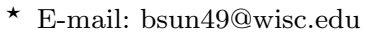

$\dagger$ E-mail: orio@astro.wisc.edu
}

cle of classical and recurrent novae $(\mathrm{CN}, \mathrm{RN})$ are mostly determined by two fundamental parameters: the white dwarf (WD) mass and the mass transfer rate $\dot{m}$ onto the WD (Starrfield et al. 2012; Wolf et al. 2013). For simplicity, most numerical models of novae outbursts involve a constant $\dot{m}$; however, there is no reason for which $\dot{m}$ should not vary. The so-called "hibernation model" predicts short periods of high $\dot{m}$ before and after nova outbursts, when the nuclear burning WD irradiates the secondary so that mass transfer is enhanced (see Shara et al. 1986; Hillman et al. 2020, the latter an updated version of the model). $\dot{m}$ may also vary less regularly, due to interactions with the secondary (Shaviv 2017).

The observations analyzed here were part of a long term project of following novae with well-known outburst characteristics and parameters, as they settle into quiescent accretion. The most recent previous articles were part of Polina Zemko's thesis at the University of Padova (Zemko et al. 2015, 
2016), and were preceded by several other observations with X-ray telescopes by Orio, her group, and collaborators (Orio 1993; Balman et al. 1995; Orio et al. 1996; Mukai \& Orio 2005; Orio et al. 2009; Nelson et al. 2011; Mason et al. 2013; Zemko et al. 2015, 2016, 2018). The X-ray range is particularly interesting to study quiescent novae and all other cataclysmic variables (CVs). In disk accretors, by converting gravitational into electromagnetic energy, $\dot{m}$ powers the X-ray luminosity of the disk boundary layer. Thus, $\dot{m}$ can be estimated with a model fit, of plasma in collisional ionization equilibrium, often with a gradient of plasma temperature (see Mukai et al. 2003). Measuring and fitting the complete X-ray spectrum is fundamental for this aim. The hot plasma's maximum temperature is also directly related to the WD mass because the temperature of the shock is directly proportional to the potential well of the WD. In magnetic systems, where accretion is funneled to the magnetic poles of the WD, a strong standing shock is formed near the WD surface. Thus, the X-ray flux and spectrum trace the accretion process and its rate, which gives an estimate of the WD mass. By and large, the X-ray emission of CVs correlates well with theoretical expectations (e.g. Pandel et al. 2005).

Of course even at quiescence, CVs are complex systems with several components that emit in different wavelength ranges: the accreting $\mathrm{WD}$, the mass-loosing main-sequence (or slightly evolved) secondary, the accretion disk and/or the accretion stream to the poles (for highly magnetized WDs) and winds or other outflows from the binary. Therefore, the physics inferred from the X-ray observation varies from one system to another. At high accretion rates, the boundary layer may not remain optically thin and the the accretion disk may be flared, forming a corona that emits mainly at far UV wavelengths rather than at the expected X-rays wavelength (Mauche 1998). Moreover, outflows from the disk may also contribute to the X-rays (e.g. Nixon \& Pringle 2020).

In addition to the possibility of estimating $\dot{m}$, its variations, and the WD mass, one particularly interesting problem onto which X-ray observations offer a window is the effect of the WD magnetic field on nova outbursts and evolution. The magnetic field is not a simple variable to take into account in nova models, but it may be a fundamental one. We have found evidence that some novae are intermediate polars (Ps, see Zemko et al. 2016; Aydi et al. 2018; Zemko et al. 2018). IPs host WDs whose magnetic field strengths reach several $10^{5}$ Gauss. An accretion disk forms, but it is truncated where the ram pressure of the matter in the disk is equal to the magnetic pressure of the WD's magnetic field (at the the Alfvèn radius) and the matter is channeled to the poles. WDs in IPs are not synchronized with the orbital period like the more magnetized polars, and since the magnetic axes of WDs are generally inclined with respect to their rotation axes, the asynchronous primary is an oblique rotator. X-ray flux modulation with the WD spin period is one of the main observational properties of IPs. Modulations due to this WD spin, with a period of tens of minutes are typically detected in the X-ray light curves of IP. Measuring the WD spin in X-rays is considered the best indirect proof of the IP nature; in fact, given the range of magnetic field $\left(10^{5}-10^{6}\right.$ Gauss) and the distances of known IPs, in many cases direct measurements of circular polarization only yield upper limits for the magnetic field. At distances of a few Kpc, the indirect measurement is very often the only feasible one (see Ferrario et al. 2015, for a review).

\section{TARGETS OF THESE OBSERVATIONS}

The first three observations were done with XMM-Newton, and we present here the broadband X-ray spectra taken with EPIC. The Reflection Grating Spectrographs, or RGS, did not collect enough photons; we obtained some optical and ultraviolet photometric measurements with the Optical Monitor, that we do not present here. The range of EPIC pn is 0.15-12 keV, but we had a large background in the softest band, so we limited the spectral analysis to above $0.2 \mathrm{keV}$. There is also an intense complex of detector-intrinsic lines due to $\mathrm{Cu}-\mathrm{K} \alpha, \mathrm{Ni}-\mathrm{K} \alpha$ and $\mathrm{Z}-\mathrm{K} \alpha$ lines around $8 \mathrm{keV}$, so we preferred to fit the pn spectrum below $8.0 \mathrm{keV}$ for V2491 Cyg. The MOS's are calibrated at $0.3-12 \mathrm{keV}$. For both cameras, due to the very low counts above $10 \mathrm{keV}$, we extracted the data with $10 \mathrm{keV}$ as upper limits. The $0.2-10 \mathrm{keV}$ count rates we detected with the EPIC cameras and the observation dates and exposure times are in Table 1 . We could not use all the exposure time due to high background intervals, that we removed to obtain "clean" spectra. KT Eri was also observed with the ACIS-S camera of Chandra. The last observation, V794 Aql, was done with Chandra, the ACIS-S camera, and the High Energy Transmission Gratings (HETG).

\subsection{V2491 Cyg}

V2491 Cyg had a luminous nova outburst in 2008 April, reaching $\mathrm{V}=7.7$ (Nakano \& Sumoto 2008), with an amplitude of 9.4-mag in the V band (Jurdana-Sepic \& Munari 2008). It was a fast nova, with $t_{2}$ (time for a decrease by 2 -mag in optical) of 4.6 days, and the ejecta velocity reached $4860 \mathrm{~km}$ $\mathrm{s}^{-1}$. A rebrightening occurred at the end of April, then the luminosity declined to $\mathrm{V} \simeq 16$ after about 150 days (1 mag brighter than pre-outburst level). The orbital period is not known, although a modulation with a period of $\simeq 2.3$ hours detected in outburst (Baklanov et al. 2008) was proposed to be orbital in nature. No periodicity, however, was detected in quiescence by Shugarov et al. (2010). The distance to the nova is not known, because the Gaia parallax was not determined, although estimates were proposed, $10.5 \mathrm{kpc}$ (Helton et al. 2008) and $14 \mathrm{kpc}$ (Munari et al. 2011), based on the Maximum Magnitude versus Rate of Decline (MMRD) relation. The optical spectra of V2491 Cyg are similar to two known RN, U Sco and V394 Cra (Tomov et al. 2008), although there is no evidence, so far, of a previous outburst of this nova.

In the outburst, V2491 Cyg became a very luminous supersoft X-ray source (SSS) when the central source contracted, and the ejecta became transparent to supersoft X-rays, between day 36 and 42 (Page et al. 2010). The SSS flux increased until day 57 and then started decreasing. As the X-ray flux decreased, the temperature seemed to remain constant (Page et al. 2010), indicating a possible shrinking of the emitting region rather than cooling. This resembles the cases of V407 Lup (Aydi et al. 2018) and V4743 Sgr (Zemko et al. 2016), two novae that are thought to be IPs, in which the nuclear burning seemed to last longer in a restricted region of the WD surface, presumably at the polar caps where accretion 
Table 1. Details of the XMM-Newton EPIC observations. The count rates are corrected for the background, and are in the $0.2-10$ keV range for the pn and 0.3-10 keV for the MOS. The exposure length is the nominal exposure (individual MOS or pn exposures may be shorter by up to $2 \mathrm{ks}$. The time used for EY Cyg refers only to the spectral analysis, while for the timing analysis we could use the whole interval.

\begin{tabular}{cccc}
\hline Target Name & V2491 Cyg & EY Cyg & KT Eri \\
\hline Observation Date (UT) & 2017 November 27 14:49:57 & 2007 April 23 12:43:52 & 2018 February 15 21:47:05 \\
\hline Exposure Length (ks) & 34 & 45.4 & 72.3 \\
\hline Time used (ks) & 22.2 & 20.0 & 51.1 \\
\hline MOS1 Net Count Rate $\left(\mathrm{s}^{-1}\right)$ & $0.0793_{-0.0022}^{+0.0022}$ & $0.0941_{-0.0028}^{+0.0028}$ & $0.0142_{-0.0075}^{+0.0075}$ \\
\hline MOS2 Net Count Rate $\left(\mathrm{s}^{-1}\right)$ & $0.0840_{-0.0022}^{+0.0022}$ & $0.0995_{-0.0032}^{+0.0032}$ & $0.0143_{-0.0075}^{+0.0075}$ \\
\hline pn Net Count Rate $\left(\mathrm{s}^{-1}\right)$ & $0.3168_{-0.0050}^{+0.0050}$ & $0.3385_{-0.0055}^{+0.0055}$ & $0.0510_{-0.0014}^{+0.0014}$ \\
\hline
\end{tabular}

started again. The peak atmospheric temperature was very high compared with most novae, probably close to a million K (Ness et al. 2011), indicating a massive WD. In this phase, V2491 Cyg showed irregular variability, but also a regular modulation with a 37.2-minute period (Ness et al. 2011).

V2491 Cyg is a rare case of a nova known as an X-ray source before the outburst (Ibarra et al. 2009), and for this reason, it was observed again in quiescence with Suzaku by Zemko et al. (2015). A modulation with a period of 38 minutes (close to the period of the SSS after the outburst) was detected again, but its stability was not assessed. Zemko et al. (2015) speculated that the detected period represented the spin of an IP, and they also found that the broadband X-ray spectrum was consistent with a class called "soft IPs". However, the very soft portion of the spectrum observed for V2491 Sgr was not clearly due to accretion. The still relatively large flux and supersoft spectrum indicated the possibility that nuclear burning in a restricted region around the polar caps was observed, either because it was quenched later in hot, accreting region, or because it was kept alive by newly accreted material. This is consistent with the interpretation of an emitting region still shrinking rather than cooling, as mentioned above, in the post-outburst phase. Zemko et al. (2018) performed optical photometry, discovering a 36.24-minute period, slightly shorter than the one detected in X-ray. This may be the beat of the rotational and orbital period, giving higher credibility to the hypothesis that V2491 Cyg is also an IP. If the beat period is indeed the one measured in the optical light curve, the orbital period has to be of $\sim 17$ hours (longer than the continuous observations that were possible during the nights in that study).

\subsection{KT Eri}

KT Eri is another nova for which we may connect a wellstudied outburst behavior with accretion and quiescence properties. It was discovered by Yamaoka et al. (2009) at $\mathrm{V}=8.1$, but probably not on the actual outburst day. Drake et al. (2009) suggested the outburst occurred after 2009 November 10. Hounsell et al. (2010), a pre-discovery study, suggested that the maximum was on 2009 November 14.67 at $\mathrm{V} \sim 5.42$, with a pre-outburst rise of by 3 magnitudes in 1.6 days. The outburst amplitude was $9 \mathrm{mag}$ and the estimated time for a decay by 2 magnitudes was $t_{2}=6.6$ days (Hounsell et al. 2010), indicating that KT Eri was a fast nova. The dis- tance is $3.69_{-0.43}^{+0.53} \mathrm{kpc}$, obtained thanks to the Gaia parallax 1. The ejecta velocity reached a maximum of $2800 \pm 200 \mathrm{~km}$ $\mathrm{s}^{-1}$ (Ribeiro et al. 2011). Jurdana-Šepić et al. (2012) found that at the nova at quiescence varies with two distinct periods of 376 and 737 days, and a 1 mag amplitude. These authors noted the similarity of the optical and X-ray light curve of KT Eri with those of known RNe, with which the nova also had a large ejection velocity in common, but no previous outburst could be found in the Harvard plates. Jurdana-Šepić et al. (2012) thus suggested that the outbursts recur perhaps on timescales of a few centuries. The companion is very likely to be an evolved star, ascending towards the red giant branch. In X-rays, KT Eri was first detected with the Swift XRT as an SSS on day 39.8 after its outburst (Bode et al. 2010). Later, the SSS flux extremely softened on day 65.6. The timing analysis of the Swift XRT data from day 66.60 to 79.25 revealed a $35 \mathrm{~s}$ modulation (Beardmore et al. 2010), that was later confirmed by Ness et al. (2015) with Chandra. Ness et al. (2015) showed that this modulation was detected only during the early SSS phase, and then again much later, on day 159. With the Chandra low energy grating spectrograph (LETG), the nova spectrum appeared similar to N SMC 2016 (Orio et al. 2018), with the luminous continuum and deep absorption lines that are typical of many novae X-ray spectra (Ness et al. 2010).

\subsection{EY Cyg}

EY Cyg is a dwarf nova of the U Gem type, and it is of particular interest to us because it is also a candidate old nova: a diffuse remnant was observed in an optical image by Sion et al. (2004). The outburst may have happened about 200 years ago, according to the above authors. The DN outbursts may indicate that it is currently transferring mass at a lower rate than at the time it had the nova outburst (see Shara et al. 1986; Sion et al. 2004). EY Cyg is known as a disk accretor with parameters well constrained by optical and UV observations. (Sion et al. 2004) inferred a massive white dwarf (WD) of $1.26 \mathrm{M} \odot$ and a secondary of $0.59 \mathrm{M}_{\odot}$, while Echevarría et al. (2007) estimated a WD mass of $1.10 \pm 0.09$

\footnotetext{
1 From the Gaia database using ARI's Gaia Services, see https://www2.mpia-hd.mpg.de/homes/calj/gdr2_distances/ gdr2_distances.pdf
} 
$\mathrm{M}_{\odot}$ and a secondary of $0.44 \pm 0.02 \mathrm{M}_{\odot}$. The inclination angle should be small, and Echevarría et al. (2007) constrained to between $13^{\circ}$ and $15^{\circ}$.

The Gaia parallax revealed a distance of $636 \pm 8 \mathrm{pc}^{1}$. Smith et al. (1997) found that the secondary star is in the spectral type range be $\mathrm{K} 5$-M0, in agreement with other authors, but Echevarría et al. (2007) measured the calcium lines and claimed that this classification should be revised to $\mathrm{K} 0$ or late G-type. Sion et al. (2004) modeled the accretion disk with $\dot{m} \sim 10^{-10} \mathrm{M}_{\odot} \mathrm{yr}^{-1}$ in order to fit the far-UV spectra, but this model places the source at a larger distance than the Gaia distance, by a factor of at least 1.4, implying that the adopted mass accretion rate is, most likely, only an upper limit. Echevarría et al. (2007) measured the orbital period of EY Cyg, 11.0237976 hours; the V magnitudes of the system during quiescence and its DN outbursts are $\sim 14.8$ and $\sim 11$ respectively, and the long-term AAVSO light curve revealed that the recurrence time of the DN outbursts is about 2000 days (Tovmassian et al. 2002).

EY Cyg was detected for the first time in X-rays with ROSAT by Orio \& Ogelman (1992) in the $0.2-2.2 \mathrm{keV}$ energy range. If it is a cooling $\mathrm{CN}$ a few hundred years after the outburst, it is interesting to compare it with $\mathrm{CN}$ a few years only after the outburst.

\subsection{V794 Aql}

V794 Aql is a VY Scl type nova-like system (see Zemko et al. 2014), studied by Godon et al. (2007)), who inferred a white dwarf mass of $0.9 \mathrm{M}_{\odot}$. The distance obtained with the Gaia parallax is $641 \pm 21 \mathrm{pc}^{1}$. VY Scl binaries have occasional "low states" of lower optical and UV luminosity, during which mass transfer seems to be halted (see Zemko et al. 2014, and references therein). From an optical luminosity level between magnitude 14 and 15, V794 Aql plunges to magnitude between 18 and 20 in the low optical state. The accepted scenario is that all VY Scl in their more common high state must have high mass accretion rate, of the order of $10^{-8} \mathrm{M}_{\odot} \mathrm{yr}^{-1}$, and Godon et al. (2007) found indeed $\dot{m}=10^{-8.5}-10^{-8} \mathrm{M}_{\odot} \mathrm{yr}^{-1}$. We note that Zemko et al. (2014) estimated only a few $10^{-11}$ $\mathrm{M}_{\odot} \mathrm{yr}^{-1}$ for the VY Scl TT Ari in the high state. Perhaps $\dot{m}$ is variable even during the high states: if it is constantly high, it is not understood why it does not lead to thermonuclear burning (Zemko et al. 2014). Contrarily to an initial hypothesis of supersoft X-ray luminosity raising for nuclear burning during the low states, Zemko et al. (2014) found instead that the X-ray luminosity decreases with the optically low states in V794 Aql and in other VY Scl systems. In all examined cases, there was no luminous supersoft X-ray source witnessing nuclear burning during the low states. Zemko's 2017 Ph.D. thesis also showed that the lack of evidence of nuclear burning can either be explained with a WD whose mass is significantly lower than the Chandrasekhar mass, and/or by low time-averaged accretion rate. With either of these characteristics, VY Scl are not type Ia supernova candidate progenitors, as previously proposed, and significantly differ from $\mathrm{RNe}$, many of which seem to have a mass accretion rate of the order of $10^{-8} \mathrm{M}_{\odot} \mathrm{yr}^{-1}$. The $\mathrm{RNe}$ are not a numerous group, and several of them are symbiotics rather than CVs. In symbiotics, it is much more challenging to trace accretion via X-rays observations, for several reasons, including more absorbing material in the symbiotic nebula, and the fact that the Roche lobe is often only partially filled.

\section{SPECTRAL AND TIMING ANALYSIS OF THE OBSERVATIONS}

\subsection{V2491 Cyg: spectral analysis}

A 34 ks observation of V2491 Cyg with XMM-Newton (Observation ID: 084580101) was proposed by PI Orio and was done on 2017 November 27, 3519 days after the observed optical maximum. We obtained $22.2 \mathrm{ks}$ of good exposure time, not affected by elevated background flares. The observations were done with the thin filter; the pn was in large window mode, the MOS1 in partial large window mode ("prime W3"), the MOS2 in large window mode. The RGS spectra did not have sufficient signal-to-noise; we only analyze the EPIC Xray data here.

Fig. 1 shows the comparison of the Suzaku spectrum observed on November 20 2011. The EPIC pn is sensitive at lower energy than Suzaku and has a large effective area at very soft energy, while Suzaku is more sensitive than EPIC around 6.5-7 keV, where the main iron lines are observed. Despite these differences, we established that the 2011 spectrum was somewhat harder. Even if the iron lines are not well resolved with EPIC, it seems the iron reflection line at $6.4 \mathrm{keV}$ of neutral iron was less intense in 2017 because, in the EPIC spectra, the iron complex seems to had been dominated by the H-like and He-like lines (Fe XXVI and Fe XXV).

Two different models fit the XMM-Newton spectra of V2491 Cyg. Only one spectral component does not fit the whole spectrum; the less complex model we could use (Model 1 in Table 2) includes two absorbed components: a blackbody and a thermal plasma in collisional ionization equilibrium, calculated with the Astrophysical Plasma Emission Code (APEC) in XSPEC (Smith et al. 2001). We modeled the interstellar absorption with the Tübingen-Boulder TBABS model (Wilms et al. 2000). The plasma temperature is not constrained in this fit and within $90 \%$ probability it may be as high as the maximum value $(64 \mathrm{keV})$ of the model; so we fixed it at $10 \mathrm{keV}$, close to the Suzaku temperature, to constrain at least the other parameters. The second model (Model 2 in Table 2) is the one that fitted the Suzaku data in work by Zemko et al. (2015); it includes a second thermal component at a very low temperature, and for the hotter plasma, a partially covering absorber, which is often used to fit IP spectra, because of the accretion column partially obscuring the emission. This model also includes a Gaussian to fit the iron complex. Although there is a need of a separate line for the reflection line of iron at $6.4 \mathrm{keV}$, it is not clearly resolved from the iron Fe XXV triplet around $6.7 \mathrm{keV}$ that, in the XMM data, it appears stronger than the line at $6.4 \mathrm{keV}$. Perhaps this is due to enhanced iron with respect to the solar value, but we did not have enough signal to noise and resolution to assess this. The absorption is modeled with WABS, the Wisconsin "recipe" (Morrison \& McCammon 1983), not with TBABS, as in our models in this paper. The fit parameters are in Table 2. The EPIC-pn spectrum and the fit with Model 2 are shown in Fig. 1 together, for comparison, with the Suzaku spectrum and fit by Zemko et al. (2015); the three EPIC spectra fitted with Model 1 are shown in Figure 2. 
Table 2. Best fit parameters for the V2491 Cyg combined EPIC spectra with Model 1 (using TBABS) and Model 2 (using WABS, and the plasma temperature of the best fit by the above authors for Suzaku). $L_{b b}$ is the bolometric luminosity of the blackbody, assuming a distance of $10.5 \mathrm{kpc} . F_{\mathrm{abs}}$ and $F_{\text {unabs }}$ the absorbed and unabsorbed X-ray flux, in different energy ranges. Errors are estimated at the $90 \%$ confidence level.

\begin{tabular}{|c|c|c|c|}
\hline Parameter/Result & Model 1 & Model 2 & Suzaku (Zemko et al. 2015) \\
\hline $\mathrm{N}(\mathrm{H})\left(\right.$ wabs, $\left.\times 10^{22} \mathrm{~cm}^{-2}\right)$ & $0.40_{-0.04}^{+0.05}$ & $0.30_{-0.02}^{+0.04}$ & $0.25_{-0.06}^{+0.08}$ \\
\hline Blackbody Temperature (eV) & $76_{-3}^{+3}$ & $82_{-7}^{+3}$ & $77_{-9}^{+7}$ \\
\hline$L_{b b}\left(\times 10^{35} \mathrm{erg} \mathrm{s}^{-1}\right)$ & $2.77_{-0.98}^{+1.62}$ & $1.03_{-0.37}^{+0.61}$ & $1.4_{-0.7}^{+2.4}$ \\
\hline $\mathrm{N}(\mathrm{H})\left(\right.$ pcfabs, $\left.\times 10^{22} \mathrm{~cm}^{-2}\right)$ & - & $14.9_{-4.0}^{+5.0}$ & $13.3_{-2.4}^{+3.0}$ \\
\hline Partial Covering Fraction & - & $0.57_{-0.07}^{+0.06}$ & $0.66_{-0.03}^{+0.03}$ \\
\hline$T_{1}(\mathrm{keV})$ & - & 0.24 (Fixed) & $0.24_{-0.24}^{+0.24}$ \\
\hline$T_{2}(\mathrm{keV})$ & $10.0($ Fixed $)$ & 11.3 (Fixed) & $11.3_{-1.5}^{+1.8}$ \\
\hline $\operatorname{Norm}_{1}\left(\times 10^{-4} \mathrm{~cm}^{-5}\right)$ & - & $0.0_{-0.0}^{+2.2}$ & $5_{-5}^{+8}$ \\
\hline $\operatorname{Norm}_{2}\left(\times 10^{-4} \mathrm{~cm}^{-5}\right)$ & $5.5_{-0.6}^{+0.6}$ & $11.7_{-0.9}^{+2.0}$ & $14.6_{-1.0}^{+1.1}$ \\
\hline Gaussian EW (Fixed) (eV) & 245 & 245 & 245 \\
\hline$\chi^{2}$ & 1.6 & 1.1 & 1.1 \\
\hline$F_{\mathrm{abs}, 0.2-1.0 \mathrm{keV}}\left(\times 10^{-13} \mathrm{erg} \mathrm{cm}^{-2} \mathrm{~s}^{-1}\right)$ & $\begin{array}{r}2.43_{-0.34}^{+0.04} \\
\end{array}$ & $2.44_{-0.27}^{+0.37}$ & 4.38 \\
\hline$F_{\mathrm{abs}, 0.2-10.0 \mathrm{keV}}\left(\times 10^{-12} \mathrm{erg} \mathrm{cm}^{-2} \mathrm{~s}^{-1}\right)$ & $1.40_{-0.08}^{+0.04}$ & $1.64_{-0.07}^{+0.07}$ & 2.10 \\
\hline$F_{\text {unabs, } 0.2-1.0 \mathrm{keV}}\left(\times 10^{-12} \mathrm{erg} \mathrm{cm}^{-2} \mathrm{~s}^{-1}\right)$ & 14.71 & 5.84 & 7.98 \\
\hline$F_{\text {unabs, } 0.2-10.0 \mathrm{keV}}\left(\times 10^{-12} \mathrm{erg} \mathrm{cm}^{-2} \mathrm{~s}^{-1}\right)$ & 15.98 & 7.31 & 9.71 \\
\hline
\end{tabular}

V2491 Cyg Spectra: XMM-Newton pn VS. Suzaku FI

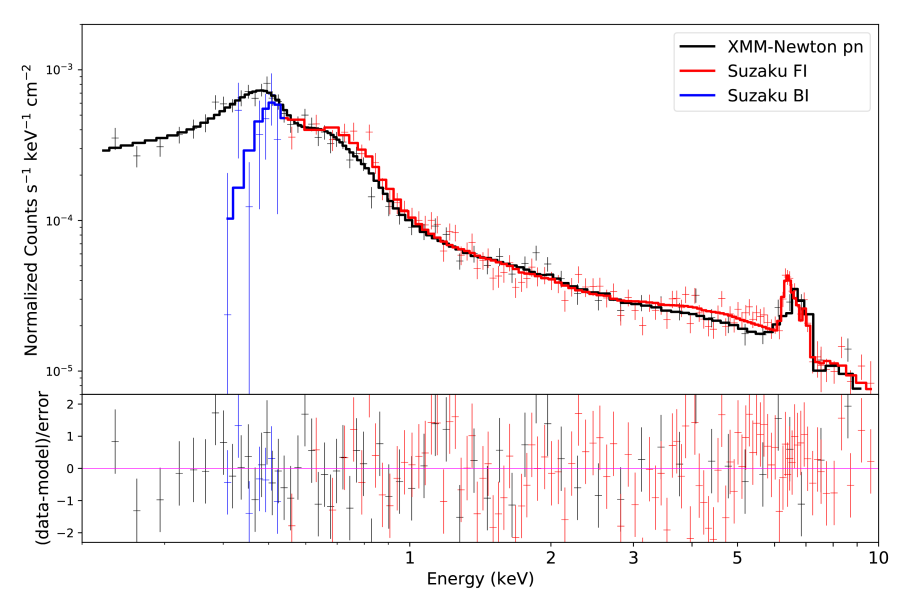

Figure 1. V2491 Cyg EPIC pn spectrum in black and Suzaku FI (front illuminated) CCD spectrum (in red) and the soft portion of the BI (back illuminated) spectrum in blue. The best fit with the model in the second and third column of Table 1 (the model of Zemko et al. (2015) for the Suzaku spectrum and its application to the EPIC pn one, are also shown.

The results indicate a significant change (about $44 \%$ in the best fit) in the unabsorbed flux and by about $22 \%$ in the unabsorbed flux; this is due mostly to the flux between 0.7 and $1 \mathrm{keV}$ as it is evident in Fig.1. We plotted here the spectrum of the FI (front-illuminated) CCD above $0.8 \mathrm{keV}$, because it

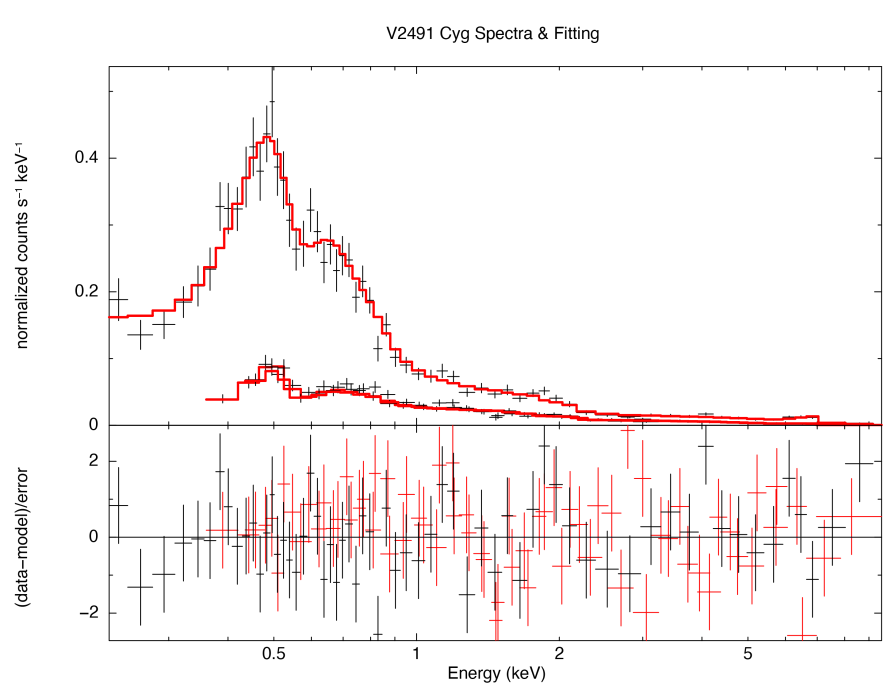

Figure 2. V2491 Cyg EPIC spectra in units of count rate. The pn data are the higher ones, MOS are the lower values. The best fit with Model 2 is in red. The residuals for the pn are in black and for the MOS in red.

has the best signal-to-noise, but, additionally, below $0.8 \mathrm{keV}$ we plotted the spectrum of the BI (back-illuminated) CCD that is sensitive and calibrated at softer energy. The blackbody component may not have varied much, but the Suzaku instruments did not constrain it as well as EPIC did. On the other hand, the Suzaku cameras were much more sensitive to 
the 6.4-7.0 keV range in which the iron complex is measured, giving other diagnostics. We find that the unabsorbed flux in the $0.7-1 \mathrm{keV}$ range has decreased by $45 \%$ since the Suzaku observation, and the unabsorbed one by up to $25 \%$, as Fig. 1 shows. On the other hand, with XMM-Newton we detected copious blackbody-like X-ray flux below $0.8 \mathrm{keV}$, corresponding to a luminosity of $2.77 \times 10^{35} \mathrm{erg} \mathrm{s}^{-1}$ when we account for the absorbing column and assume a $10.5 \mathrm{kpc}$ distance.

We follow Patterson \& Raymond (1985) and other authors, in estimating $\dot{m}$ by assuming that about half of the gravitational energy due to accretion is converted in X-ray luminosity at the boundary layer. Those authors considered the unabsorbed X-ray luminosity in the $0.2-4.0 \mathrm{keV}$ range and made the assumption that it is about a quarter of the X-ray luminosity at all wavelengths. Of course, this depends on the slope of the spectrum. We estimated the unabsorbed X-ray flux in the $0.2-10 \mathrm{keV}$ range and since V2491 Cyg does have a hard tail, we extrapolated the model flux at hard energy 10-100 keV, but found it may represent only about $5 \%$ of the unabsorbed X-ray flux in the $10-100 \mathrm{keV}$ range. After a simple manipulation the resulting formula, equivalent to rescaling Equation No.4 of the above authors, we obtained

$\dot{M}_{16}=\frac{L_{x}(0.2-10.0 \mathrm{keV})}{33.0 \times 10^{31} M_{0.7}^{1.8}} g \mathrm{~s}^{-1}$

where $\dot{M}_{16}$ is the mass accretion rate in units of $10^{16} \mathrm{~g} \mathrm{~s}^{-1}$. If we include all the X-ray flux, including the blackbody component, the result is $\dot{m}=1.26 \times 10^{18} \mathrm{~g} \mathrm{~s}^{-1}=1.97 \times 10^{-8} \mathrm{M}_{\odot} \mathrm{yr}^{-1}$ (assuming a $10.5 \mathrm{kpc}$ distance), comparable with the estimate for RNe. If the blackbody-like flux is not associated with accretion and it is still atmospheric, it contributes to a large fraction of the total flux. In fact, the bolometric luminosity of the blackbody model is of the same order of magnitude as the total X-ray flux for a $10.5 \mathrm{kpc}$ distance. As hypothesized and suggested by Zemko et al. (2015), we may instead be still observing a very hot stellar atmosphere, which is about 10 times less luminous than a blackbody at the same temperature (see the alternative fit with the atmosphere in that paper). In this case, we need to exclude this large, soft portion of the X-ray flux in accounting for luminosity due to accretion. For the sake of simplicity, we excluded all unabsorbed flux below $1 \mathrm{keV}$ (this includes more than the blackbody-like component's contribution) and found that the unabsorbed Xray flux is $1.36 \times 10^{-12} \mathrm{erg} \mathrm{cm}^{-2} \mathrm{~s}^{1}$, which indicates $\dot{m}$ of order $10^{-9} \mathrm{M}_{\odot} \mathrm{yr}^{-1}$ at $10.5 \mathrm{kpc}$ distance. We thus estimate the $\dot{m}$ value to be between this and the above value. We note that $\dot{m}$ scales with the square of the distance ratio, so at $14 \mathrm{kpc}$ the range would increase by a factor of 1.78 .

\subsection{V2491 Cyg: timing analysis}

The Lomb-Scargle periodogram (Scargle 1982) of the EPIC pn light curve, without any de-trending, is shown in Fig. 3. Here and in the following analysis, we calculate the statistical error of the frequency as half-width at half amplitude. The periodogram is dominated by two peaks, $f_{1}$ and $f_{2}$. The first frequency has power below the $90 \%$ confidence range, while the second one has power above the $99.9 \%$ confidence range. $f_{2}$ is the known $\simeq 38$ min periodicity, We did not detect any frequency with high power in the MOS1 light curve, but we did detect the frequency $0.432_{0.020}^{+0.021} \mathrm{mHz}$ (corresponding to $f_{2}$ and to a period of 38.6 minutes) with a significance of $99 \%$ in the MOS2 data. This frequency value is within the error bar of the pn measured $f_{2}$. We will continue the discussion referring to the EPIC pn, since it offers a higher S/N. After detrending the EPIC pn light curve with a $4^{\text {th }}$ order polynomial, also the $f_{1}$ peak became significant, just slightly below the $99 \%$ confidence level (top panel of Fig. 4). We experimented by splitting the detrended light curve into two halves, and found that both peaks remained dominant in both periodograms (middle and bottom panel of Fig. 4), but the significance for both peaks dropped considerably below the $90 \%$ confidence level in the first half, while $f_{2}$ was detected at a high confidence level in the second half. All the values of the measured frequency are listed in Table 3.2. The binned light curves are plotted in phase using $f_{1}$ and $f_{2}$ in Fig. 5. We note the double-humped structure in the $f_{1}$ plot.

Even if the frequencies detected in this work do not show any significant change after splitting the light curve into two equal segments, which suggests stability, the errors are too large for a conclusive statement on the stability of the modulations. However, first of all, we confirm the previously detected period near $38 \mathrm{~min}$. Second of all, the exact value derived from our XMM-Newton EPIC-pn data is slightly longer, 39.6 min and Fig. 4 suggests a possible continuous shift of the periodicity toward longer periods, but the measurement uncertainties in all three epochs do not allow us to make a definite statement. All the measured values at different epochs are within each other's errors.

The third important point is the following: the detection of $f_{1}$, which is very close to half $f_{2}$, implies that $f_{2}$ may be the first harmonic of $f_{1}$. The confidence of this pattern in the two subsamples (the light curve split into two halves), after de-trending, is below $90 \%$, which suggests that this pattern must be taken with caution. If it is real, the fundamental frequency may be $f_{1}$, while $f_{2}$ is its first harmonic. The detected periodicity of $39.6 \mathrm{~min}$ would be (within the errors) half of the white dwarf rotation period. The frequency $f_{1}=0.205 \mathrm{mHz}$ yields a value of the period of $81.3 \mathrm{~min}$.

In order to assess the energy dependence of the variability, we extracted four light curves in different energy bands: 5$10 \mathrm{keV}$ (band 1), 3-5 keV (band 2), 0.8-3 keV (band 3) and $0.3-0.8 \mathrm{keV}$ (band 4 ). The count rates in each band are 0.03 $\operatorname{cts~s}^{-1}$ in band 1 and $2,0.10 \operatorname{cts~s}^{-1}$ in band $3,0.17$ cts s$^{-1}$ in band 4 . Because of the lower count rate in each narrow band, the power of the detected periodogram peak decreases, but we compared the corresponding periodograms with the 0.3-8 keV light curve in Fig. 6. After accounting for the different average count rates, we still agree with the conclusion of Zemko et al. (2015) that the largest modulation is at 0.8-3 $\mathrm{keV}$, although it is almost comparable even at $3-5 \mathrm{keV}$ (and is hardly measurable in band 1 , that has the same count rate).

\subsection{The KT Eri X-ray spectra}

When the nova returned to quiescence, we examined an archival Chandra ACIS-S observation (PI Fred Walter) done on 2011 November 13, almost exactly 3 years after the eruption (day 1094 after the inferred optical maximum). The energy range is $0.3-10 \mathrm{keV}$. This exposure lasted for $75 \mathrm{ks}$ revealed an intriguing soft portion of the spectrum of KT Eri in quiescence, which resembled the first post-outburst quiescent X-ray spectra of V4743 Sgr and V2491 Cyg. The Chandra 


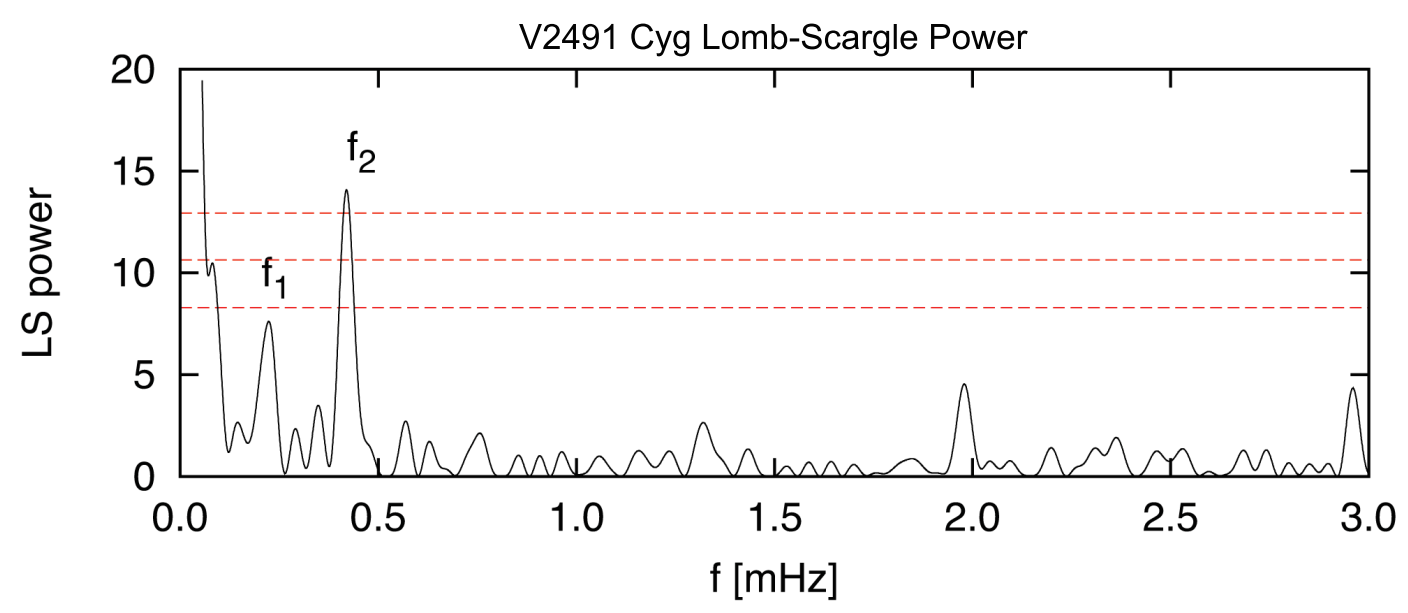

Figure 3. Periodogram of non-de-trended EPIC pn light curve of V2491 Cyg. The red lines represent the 99.9\%, 99.0\% and 90.0\% confidence levels.

Table 3. Frequencies in the EPIC pn and Suzaku light curves of V2491 Cyg, and days after the outburst optical maximum.

\begin{tabular}{llccc}
\hline day & curve portion & $\begin{array}{c}f_{1} \\
(\mathrm{mHz})\end{array}$ & $\begin{array}{c}f_{2} \\
(\mathrm{mHz})\end{array}$ & Reference \\
\hline 40 & pn, post-dip portion & & $0.448 \pm 0.036$ & Ness et al. (2011) \\
\hline 937 & pn, all & & $0.434_{-0.002}^{+0.003}$ & Zemko et al. (2014) \\
\hline 3519 & pn, all & $0.205_{-0.024}^{+0.027}$ & $0.421_{-0.021}^{+0.022}$ & this work \\
\hline 3519 & pn, first half & $0.189_{-0.048}^{+0.069}$ & $0.401_{-0.038}^{+0.041}$ & this work \\
\hline 3519 & pn, second half & $0.224_{0.055}^{0.051}$ & $0.424_{-0.050}^{0.043}$ & this work \\
\hline 3519 & MOS2, all & & $0.432^{+0.021}++_{-0.020}$ & this work \\
\hline
\end{tabular}

spectrum and the best fit to it are shown in Fig. 7 and in Table 4 . Although the fit is statistically acceptable, the residuals around $0.6 \mathrm{keV}$ with a solar abundance model may be due to enhanced oxygen.

The nova was observed again by us (PI Orio) with XMMNewton (Obs. ID: 0804580201) on February 15, 2018, on day 3378 after the inferred optical maximum. The observations were done with the thin filter, the pn and the MOS2 were used in full window mode and MOS1 in large window mode. The nominal exposure of $72.3 \mathrm{ks}$ was partially affected by a very elevated background, so we used $51.1 \mathrm{ks}$ of good exposure time.

Fig. 7 shows also the comparison between the Chandra exposure and the XMM-Newton one. The spectrum became harder during the intervening years.

We fitted the three EPIC spectra simultaneously. The fit with Model 2 is shown in Figure 8 and the fit parameters are in Table 4 . In both the XMM-Newton and the Chandra observations, an APEC low-temperature thermal component of plasma in collisional ionization equilibrium fits the softest portion of the spectrum a little better than a blackbody. This soft flux decreased by more than half between the time of the Chandra and the XMM-Newton observation, but the decrease is explained by the model as due to higher absorption (we can only speculate that it may have been intrinsic and due to wind from the disk). In any case, the unabsorbed luminosity of this component is about $2 \times 10^{32} \mathrm{erg} \mathrm{s}^{-1}$, three orders of magnitude less than for V2491 Cyg. Another plasma component is added for Chandra in Model 1, while a power law is added in Model 2. We could not constrain the temperature of the second thermal component, which, in the XSPEC fit, became arbitrarily high, outside the EPIC range, like in Model 1, so we fixed at $10 \mathrm{keV}$. The power law provides an acceptable fit and turns out to be the dominant flux component in this fit, although a non-thermal component in a $\mathrm{CV}$ is not usually detected.

We do know that the column density to the nova is low, because of the luminous supersoft X-ray source detection; an analysis of the outburst Chandra spectra by Pei et al. (private communication) indicates with $90 \%$ confidence probability that $\mathrm{N}(\mathrm{H})<\times 10^{21} \mathrm{~cm}^{-2}$. However, we can only fit the data with column density $\mathrm{N}(\mathrm{H})=10^{21} \mathrm{~cm}^{-2}$ for the soft component and much higher column density for the hard component, because the spectrum above $1 \mathrm{keV}$ is flatter and quite different from other novae and CVs. We suggest that the system may be surrounded by a strong disk wind that causes intrinsic absorption. We also attempted introducing a partially covering absorber like for V2491 Cyg, but the resulting covering fraction would exceed $80 \%$ and thus does not seem very significant when compared to much smaller values obtained for known IPs.

The X-ray luminosity of KT Eri, at about $3.7 \mathrm{kpc}$ distance, 


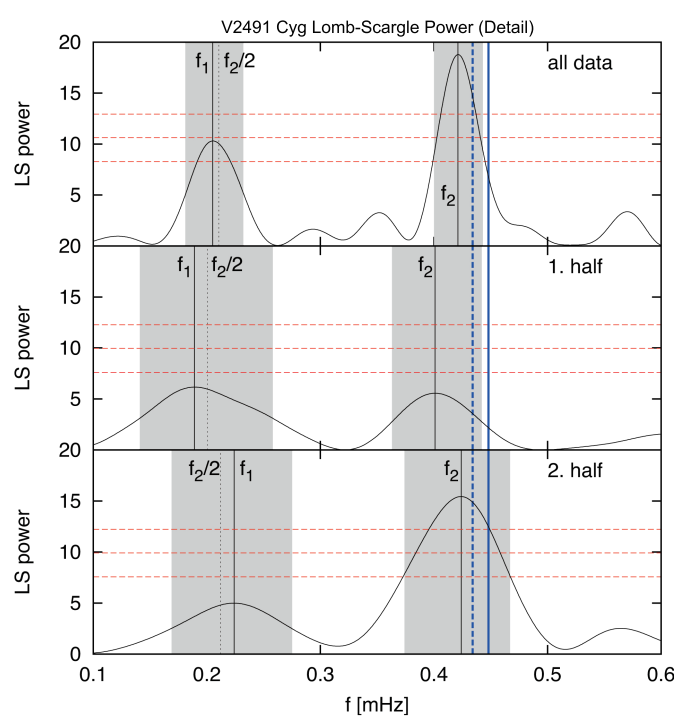

Figure 4. Periodograms calculated with the de-trended EPIC pn on the light curve of V2491 Cyg. The vertical black solid lines represent the detected frequencies, with error intervals represented by shaded areas. The vertical, dashed black lines show the $f_{2} / 2$ values. The blue lines indicate the frequencies detected in two previous observations, on day 40 after the outburst (solid line, (Ness et al. 2011)), on day and 937 (dashed line, Zemko et al. 2015)). The red lines indicate the $99.9 \%, 99.0 \%$ and $90.0 \%$ confidence levels.

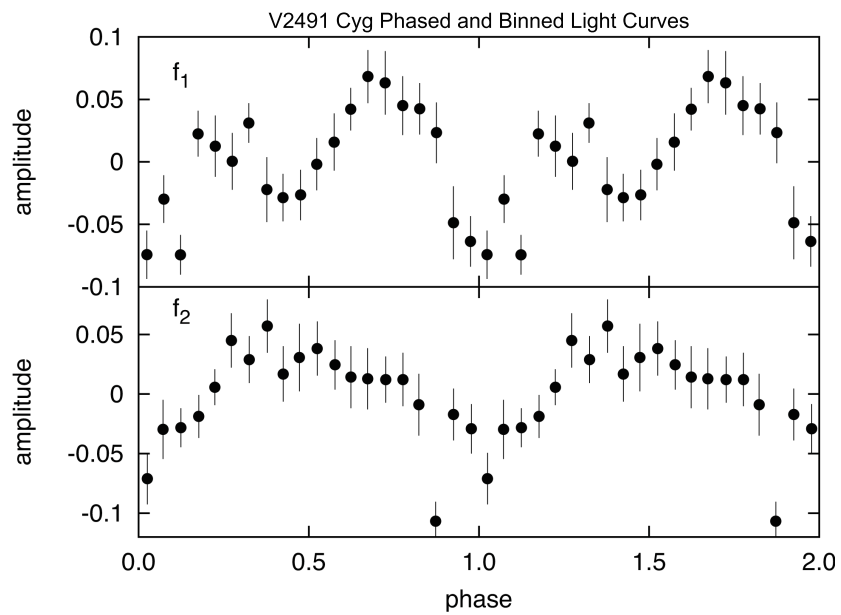

Figure 5. Phased and binned whole light curves of V2491 Cyg using two different frequencies marked as label in the upper left corner. The error-bars represent errors of the mean.

translates with Equation 1 into $\dot{m} \simeq 1.9 \times 10^{-10} \mathrm{M}_{\odot} \mathrm{yr}^{-1}$, two orders of magnitude less than V2491 Cyg.

The timing analysis did not reveal any significant periodicities, and we did not have high enough $\mathrm{S} / \mathrm{N}$ to retrieve the 35 s modulations. Such short periods, however, are typically observed only during outburst (see Ness et al. 2015).

\subsection{EY Cyg spectral analysis}

EY Cyg was observed on April 272007 (Obs. ID: 0400670101, PI Orio); the exposure lasted for each instrument, pn and MOS, lasted for $43.5 \mathrm{ks}$ (about 12.08 hours), covering the

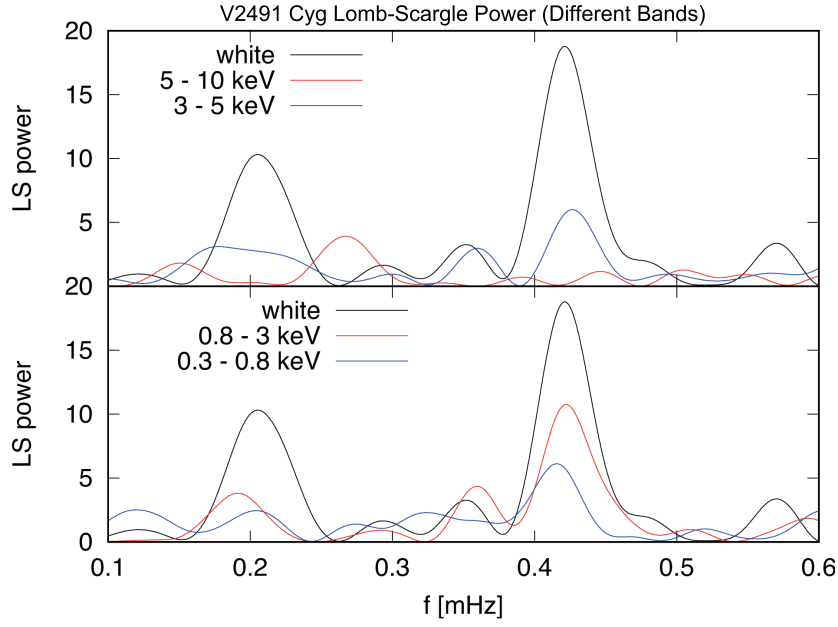

Figure 6. The same as the top panel of Fig. 4, with added periodograms obtained in different energy bands for comparison. "White" means 0.2-10 keV.

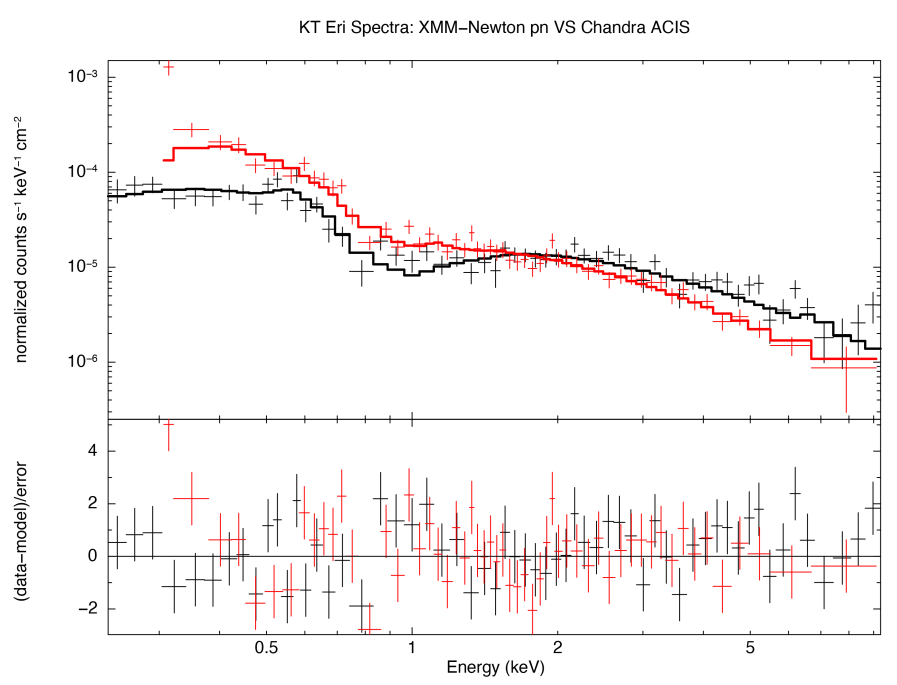

Figure 7. The Chandra ACIS spectrum of KT Eri (in red, observed in 2011 November) and the XMM-EPIC pn one (observed in 2018 February), in black. The y-axis shows the photon counts and the fits are Model 2 in Table 2, and the Suzaku model in the same table.

orbital cycle of about 11 hours about 1.1 times. The observations were done with the thin filter, the pn and the two MOS were all in full-frame mode.

Mukai et al. (2003) have argued that a cooling flow model fits well the X-ray spectra of disk accretor CVs, and we found that the XSPEC model vmcflow fits the spectra reasonably well, but so does also a two-temperature plasma model (two APEC regions). The spectrum and the spectral fit with the cooling flow is shown in Figure 9 and the best fit parameters are in Table 5.

In the cooling flow model, $\dot{m}$ is a fit parameter. We obtained the best fit with $\dot{m}=1.77 \times 10^{-11} \mathrm{M}_{\odot}$ year $^{-1}$ and the value derived from the fit with two plasma temperatures is about the same. Also the values of column density $(\mathrm{N}(\mathrm{H}))$ and the fluxes are about the same in the two different fits. 
Table 4. KT Eri Spectral Fits Parameters

\begin{tabular}{cccc}
\hline Parameter & 2 APECs & APEC + Power-law & Chandra 2 APECs \\
\hline $\mathrm{N}(\mathrm{H})_{1}\left(\times 10^{21} \mathrm{~cm}^{-2}\right)$ & $1.0_{-0.5}^{+0.7}$ & $1.0_{-0.5}^{+0.6}$ & 0.5 (Fixed) \\
\hline $\mathrm{N}(\mathrm{H})_{2}\left(\times 10^{21} \mathrm{~cm}^{-2}\right)$ & $9.4_{-1.2}^{+1.3}$ & $6.8_{-1.7}^{+1.9}$ & $5.6_{-1.9}^{+2.2}$ \\
\hline$T_{1}(\mathrm{keV})$ & $0.18_{-0.01}^{+0.02}$ & $0.18_{-0.03}^{+0.02}$ & $0.19_{-0.03}^{+0.04}$ \\
\hline$T_{2}(\mathrm{keV})$ & $10.0($ Fixed $)$ & - & $5.18_{-1.41}^{+2.37}$ \\
\hline$F_{1, \text { abs }}\left(\times 10^{-13} \mathrm{erg} \mathrm{cm}^{-2} \mathrm{~s}^{-1}\right)$ & 0.25 & 0.25 & 0.64 \\
\hline$F_{1, \text { unabs }}\left(\times 10^{-13} \mathrm{erg} \mathrm{cm}^{-2} \mathrm{~s}^{-1}\right)$ & 1.28 & 1.18 & 1.17 \\
\hline$F_{2, \text { abs }}\left(\times 10^{-13} \mathrm{erg} \mathrm{cm}^{-2} \mathrm{~s}^{-1}\right)$ & 2.85 & - & 2.77 \\
\hline$F_{2, \text { unabs }}\left(\times 10^{-13} \mathrm{erg} \mathrm{cm}^{-2} \mathrm{~s}^{-1}\right)$ & 4.33 & - & - \\
\hline $\left.\mathrm{PhoIndex}^{-13} \mathrm{erg} \mathrm{cm}^{-2} \mathrm{~s}^{-1}\right)$ & - & $1.28_{-0.16}^{+0.17}$ & - \\
\hline$F_{\text {plaw, abs }}\left(\times 10^{-13}\right.$ & 3.39 & $2.47_{-0.26}^{+0.18}$ \\
\hline$F_{\text {plaw,unabs }}\left(\times 10^{-13} \mathrm{erg} \mathrm{cm}^{-2} \mathrm{~s}^{-1}\right)$ & - & 4.43 & 3.94 \\
\hline$F_{\text {abs }}\left(\times 10^{-13} \mathrm{erg} \mathrm{cm}^{-2} \mathrm{~s}^{-1}\right)$ & $3.10_{-0.21}^{+0.16}$ & $3.64_{-0.43}^{+0.15}$ & 1.8 \\
\hline$F_{\text {unabs }}\left(\times 10^{-13} \mathrm{erg} \mathrm{cm}^{-2} \mathrm{~s}^{-1}\right)$ & 5.07 & 5.61 & 1.1 \\
\hline$\chi^{2}$ & 1.2 & & - \\
\hline
\end{tabular}

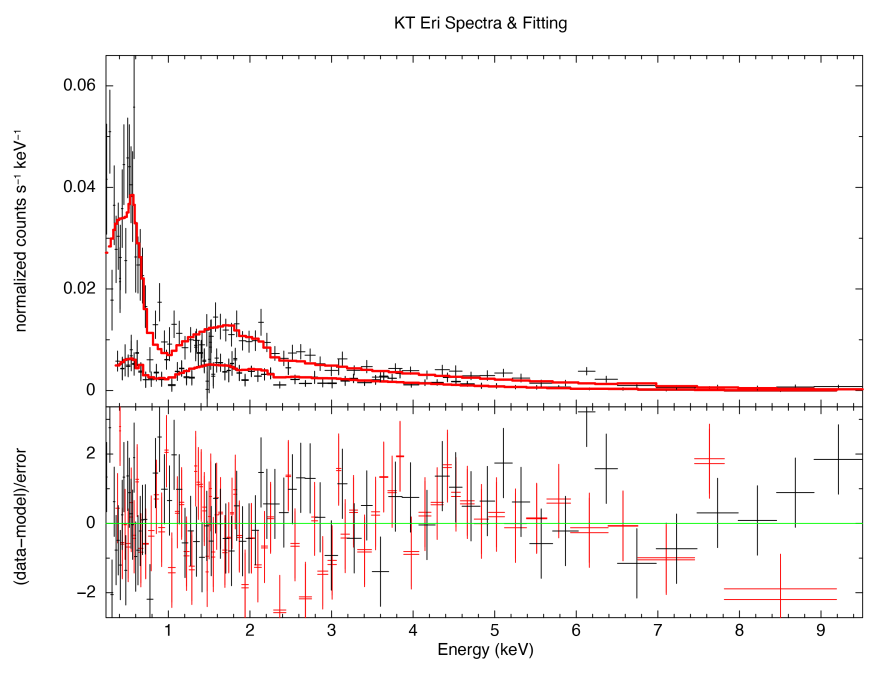

Figure 8. XMM-Newton spectra of KT Eri and the best spectral fits with Model 2. The colors are the same as in Fig. 2 and the pn again has the higher values.

\subsection{EY Cyg: timing analysis}

Because the background was enhanced, but it was not extremely elevated during much of the exposure of this target, we could correct the light curve for the background during the whole exposure and used all the exposure time of each of the three cameras. As shown in Fig. 10, in the Lomb Scargle periodogram of EY Cyg, we find a frequency of $0.205_{-0.11}^{+0.12}$ $\mathrm{mHz}$ with $99.9 \%$ probability. It corresponds to a period of $4867_{-262}^{+275} \mathrm{~s}$, or about 81 minutes, which is very different from the orbital period measured by Echevarria et al. (2007). If it corresponds to the WD rotation period, this leaves the pos-

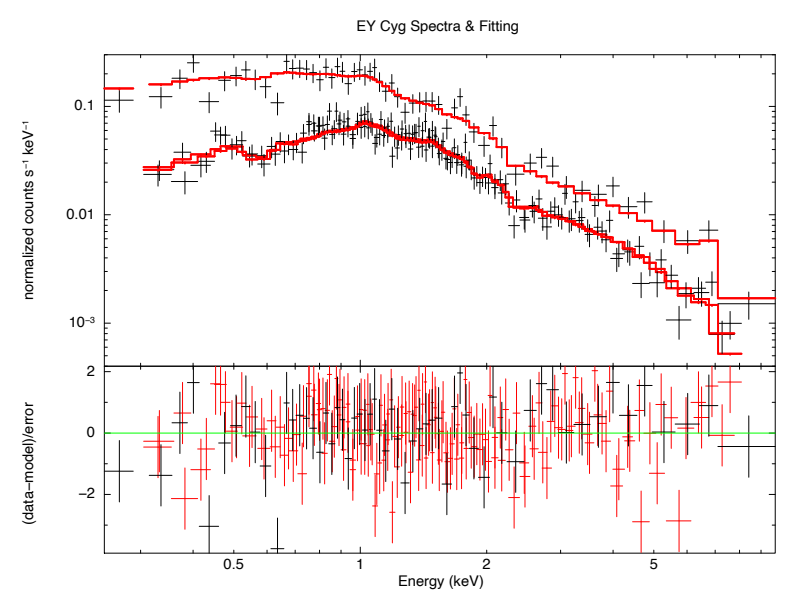

Figure 9. EY Cyg spectrum and best fit results with the model in Table 5. The pn data are above the MOS ones, the model is in red, and the residuals have the same color key as in Fig.7.

sibility open that EY Cyg is an IP like V2491 Cyg. We also show the light curve folded with this period in the second panel of Fig. 10. In the Lomb-Scargle periodogram in Fig. 10 we also notice another the frequency at $\simeq 0.4 \mathrm{mHz}$, which is close to the first harmonic of the main signal. We also experimented by splitting the light curve into two halves: in the light curve of the second half of the exposure, we found a 
Table 5. Best fit parameters for EY Cyg with a cooling flux model.

\begin{tabular}{cc}
\hline Parameter & Value \\
\hline $\mathrm{N}(\mathrm{H})\left(\mathrm{TBABS}, \times 10^{22} \mathrm{~cm}^{-2}\right)$ & $0.028_{-0.011}^{+0.012}$ \\
\hline low T $(\mathrm{keV})$ & 0.09 (Fixed) \\
\hline high T $(\mathrm{keV})$ & $23.54_{-4.8}^{+6.8}$ \\
\hline$\dot{m}\left(\times 10^{-11} \mathrm{M}_{\odot} / \mathrm{yr}\right)$ & $1.77_{-0.28}^{+0.32}$ \\
\hline$F_{\text {abs }}\left(\times 10^{-12} \mathrm{erg} / \mathrm{cm}^{2} / \mathrm{s}\right)$ & $1.34_{-0.16}^{+0.12}$ \\
\hline$F_{\text {unabs }}\left(\times 10^{-12} \mathrm{erg} / \mathrm{cm}^{2} / \mathrm{s}\right)$ & 1.44 \\
\hline$\chi^{2}$ & 1.15 \\
\hline
\end{tabular}

significant frequency at $\simeq 0.27 \mathrm{mHz}$, but it may have been a transient event ${ }^{2}$ or an artifact.

We also found an orbital modulation, as also noted by Nabizadeh \& Balman (2020), although the exposure was not sufficiently long to detect the corresponding frequency. The pn and MOS-2 light curve is shown folded over the orbital period in Figure 11. We also show the light curve in selected MOS-2 energy bands: $0.3-0.6 \mathrm{keV}, 0$ and 1-10 keV. The 1-10 $\mathrm{keV}$ band seems less modulated, and because we could only follow one orbital period, the high values may be attributed to a flux increase that is not orbital in nature. Nabizadeh \& Balman (2020) attributed the modulation mainly to the secondary star. A contribution of the disk appears unlikely at such low inclination, while it is reasonable that the modulation is indeed due to the contribution of the companion because $\mathrm{K}$ stars emit all of their X-ray flux below $1 \mathrm{keV}$ : Fig. 11 shows that the modulation is smooth at the softest energy. On the other hand, the flux below $0.6 \mathrm{keV}$ is only about $15 \%$ of the total flux and does not "pollute" the accretion disk boundary layer's flux significantly, so our conclusion on $\dot{m}$ remains valid.

\subsection{V794 Aql}

The medium energy Chandra High Energy Transmission Gratings (HETG) were used for the V794 Aql observation, specifically the Medium Energy Gratings (MEG) and the High Energy Gratings (HEG), with a respective absolute wavelength accuracy of 0.0006 and 0.011 Angstrom. The observation was done on 2015 August 25 for 94.5 kiloseconds. Because this target is not in the AAVSO or other public databases for variables, we obtained and scheduled optical observations for V794 Aql on a date as close as possible to the one of the Chandra exposure.

We monitored the target optically on 2015 August 30 of the same year with the $60 \mathrm{~cm}$ Cassegrain telescope in Stara Lesna, Slovakia, and in $\mathrm{R}$ also with the $18 \mathrm{~cm}$ Maktsutov site on the same site; then again on 2015 September 10 with the $1 \mathrm{~m}$ Cassegrain telescope at the Special Astronomical Observatory of the Caucasus in Russia. Despite some small amplitude flickering and occasional small flares, the star's lumi-

\footnotetext{
2 Dividing the light curve into many short subsamples showed this peak only in a very short time interval. However, such short light curve subsamples yield very low resolution of the corresponding periodograms, therefore not suitable for any further detailed study.
}

nosity does not vary significantly in magnitude over times of few hours, and we report here the average magnitudes in the Johnson filters. On 2020 August 30 we measured $\mathrm{R}=14.866 \pm 0.015$ in 11 exposures taken within 17 minutes, $\mathrm{V}=15.160 \pm 0.021$ in 6 exposures taken within 11 minutes, $\mathrm{B}=15.229 \pm 0.050$ in $2 \mathrm{~B}$ exposures within 3 minutes. On 2020 September 10 the average magnitudes were $R=14.627 \pm 0.012$ in 17 exposures taken within 2.86 hours, $\mathrm{V}=14.737 \pm 0.012$ in 18 exposures taken during 2.8 hours, $\mathrm{B}=14.921$ in 17 exposures taken within 2.68 hours. Honeycutt et al. (2014) showed that in the high states, V794 Aql varies between V=14 and $\mathrm{V}=15.5$, with amplitude variations within a few days; occasionally (every few years) there is a low state at $\mathrm{V} \simeq 17.5-18$, that lasts for over a month and takes a mean time of $23 \pm 4$ days for the decay to the minimum, and of $11 \pm 5$ days for the rise back to maximum. Despite the variations in the 10 days between optical observations, this interval is too long, not typical of the sharp rise observed after previous low states. The 5 days between the Chandra observation and the first optical measurement, on the other hand, would not have been enough for a full rise back to maximum. We also have to consider that no low states have been reported since 2011, and before 2007 there was a previous 11 years interval without low states (Honeycutt et al. 2014). Thus, it is extremely likely that the Chandra exposure, which is less than 5 days before the optical one was done during the more common optically high state of V794 Aql.

The Chandra data were extracted with the CIAO software (Fruscione et al. 2006) version 4.9 .1 and the CALDB calibration package version 4.8.3. The spectra were fitted with XSPEC. Unfortunately, these spectra do not have the high $\mathrm{S} / \mathrm{N}$ we had anticipated. V794 Aql was observed with about the same X-ray flux as in the low state measured by Zemko et al. (2014). Even in a previous intermediate state, the X-ray flux was four times larger. The spectrum appears to be very hard and to peak outside the Chandra ACIS+HETG range, so that we are mostly probing the low energy tail of the X-ray flux. Only emission lines at energy of at least $1.5 \mathrm{keV}(8.42$ $\AA)$ are measured. Fig. 11 shows our identification of a strong H-like line of $\mathrm{Mg}$ XII at $8.42 \AA$, while $\mathrm{Mg}$ XI He-like lines are not observed. We identified lines of S XVI, Si XIV, and Si XIII that were also strong in TT Ari (Zemko et al. 2014), and we also tentatively identify as the H-like line of Ca XX. We fitted the HEG and MEG spectra simultaneously because we had few counts per bin. Since we did not want to use a high binning factor such that would loose information on the emission lines, we used Cstat statistics (Cash 1979) to find the best fit that minimizes the Cstat parameter. Several simple models can all fit the data, albeit not perfectly: an APEC plasma in collisional ionization equilibrium, two APEC components at different temperature with solar abundance, and the MKCFLOW model described above. We show the parameter of the latter in Table 7 and the fit in Fig. 11. The maximum temperature is not well constrained and reaches the maximum value of the model, $72 \mathrm{keV}$, but at the $90 \%$ confidence level uncertainty, it may be as low as $43 \mathrm{keV}$. The predicted $\dot{m}$ is $1.5 \times 10^{-11} \mathrm{M}_{\odot} \mathrm{yr}^{-1}$. In the two APEC component models the maximum temperature also reaches the maximum value (64 keV in this case) and maybe as low as $17 \mathrm{keV}$ with the $90 \%$ confidence level uncertainty. We cannot fit the spectrum with only one temperature; in fact the 

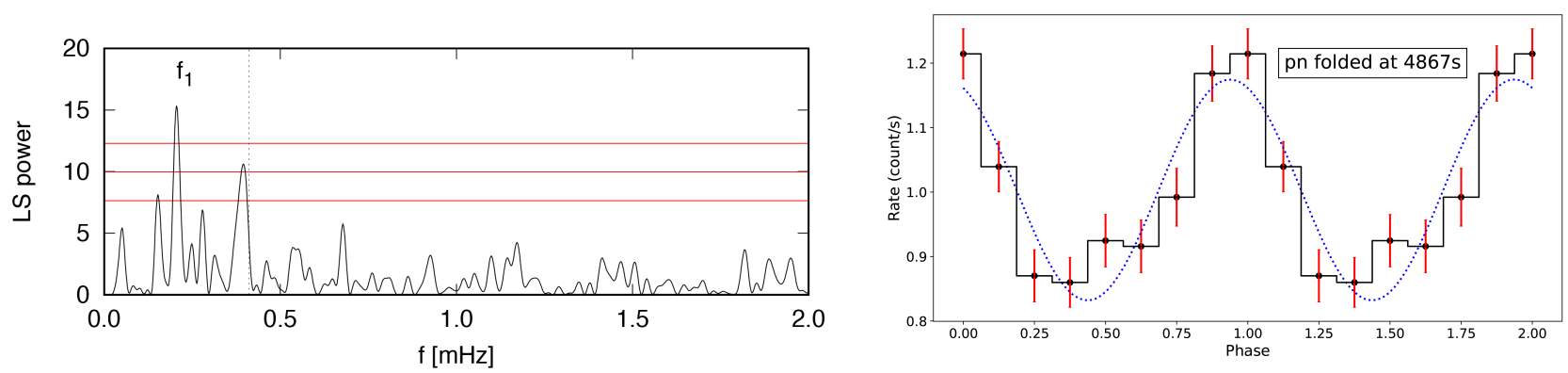

Figure 10. The first panel shows the Lomb-Scargle periodogram for the detrended pn light curve of EY Cyg in which the red lines represent the $99.9 \%, 90.0 \%$ and $80.0 \%$ confidence levels, and the vertical dashed lines shows the first harmonic of $f_{1}$. The second panel shows the pn light curve in the $0.2-10 \mathrm{keV}$ range folded with the $4860 \mathrm{~s}$ period.

Table 6. Best fit parameters for the V794Aql HEG and MEG spectra, fitted simultaneously using the MKCFLOW model in XSPEC. The maximum and minimum temperature have reached the last value included in the XSPEC model.

\begin{tabular}{cc}
\hline Parameter & Value \\
\hline $\mathrm{N}(\mathrm{H})\left(\times 10^{21} \mathrm{~cm}^{-2}\right)$ & $2.7_{-0.6}^{+1.3}$ \\
\hline $\mathrm{T}_{\text {low }}(\mathrm{keV})$ & $0.08^{+0.10}$ \\
\hline $\mathrm{T}_{\text {high }}(\mathrm{keV})$ & $72_{-39}$ \\
\hline $\mathrm{Z}$ & $1.8_{-0.8}^{+1.2}$ \\
\hline$\dot{m}$ & $2.1_{-0.3}^{1.6}$ \\
\hline $\mathrm{F}_{\text {abs. }}$ & $3.0_{-1.3}^{+2.0} \times 10^{-12}$ \\
\hline $\mathrm{F}_{\text {unabs. }}$ & $3.4 \times 10^{-12}$ \\
\hline
\end{tabular}

strong Mg XII line is produced at low temperature, and the continuum indicates a much higher temperature.

\section{DISCUSSION}

\subsection{Similar in outburst, different in quiescence}

V2491 Cyg and KT Eri share similar characteristics in being fast novae with an outburst optical amplitude of about 9 mag, at the low end for CN and closer to RN's typical amplitude. In X-rays, however, these two systems are very different at quiescence. V2491 Cyg is most likely an IP accreting at a high rate; KT Eri instead does not have signatures of a magnetic system. Assuming the reasonable hypothesis that the X-ray luminosity of the two recent novae in our sample is due to accretion, V2491 Cyg is accreting at two orders of magnitude higher rate than KT Eri. This is consistent with V2491 Cyg having a short recurrence time, perhaps as short as an RN, while either KT Eri has entered a phase of "hibernation", or its outburst recurrence time is greater than a thousand years (see model calculations of Yaron et al. 2005). The supersoft X-ray flux of V2491 Cyg was still very high at the end of 2017 and is not clearly associated with accretion. It may be due to a hot polar cap under which nuclear burning is still continuing or has just been quenched. On the other hand, the very soft portion of the KT Eri flux has much lower Xray luminosity and is consistent with accretion as underlying mechanism. Most likely, V2491 Cyg is an IP and KT Eri is a disk accretor.

\subsection{V2491 Cyg as an IP: the WD rotation period}

One of our most important findings is that a clear 39 minutes periodicity of V2491 Cyg is still measured and corresponds to the previously measured frequencies within the statistical uncertainty of the data. Thus it is very likely to be due to the WD rotation. The periodicity is not direct proof of the WD magnetic field, but it is strong evidence in favor of the classification of this nova as an IP. We also measured another frequency, about half of the known one, namely $f_{1}$. This may imply that $f_{1}$ is the true rotational frequency, while the clear detection of $f_{2}$, corresponding to the $\simeq 39$ minutes period, maybe an effect of two polar regions at different temperature and X-ray luminosity. The upper panel of Fig. 5 shows a double-humped structure, with two humps at different amplitudes. If the amplitude was almost the same, the period analysis method would "see" only the shorter period modulation $f_{2}$. One hump would be detected as one cycle, while we would not be able to identify the longer $\left(f_{1}\right)$ cycle. This may have been the case of the two previous XMM-Newton and Suzaku light curves, where the peak at $f_{1}$ did not appear. In our new data, the two humps have different amplitude, and the analysis "distinguishes" the two humps, both periodically repeated at corresponding frequency $f_{1}$, so this peak appears in the periodogram, but the folded light curve with the higher $f_{2}$ frequency does not shows this structure (lower panel of Fig. 5). A similar double-humped structure and two frequencies were detected in the X-ray observations of the IP V2069 Cyg (Butters et al. 2011), where the modulation is rotational in nature. The physical reason for V2491 Cyg may be the different luminosities of the accretion regions at the two poles, resulting in different maxima of the humps in the folded light curve. If the difference in luminosity between the two poles was not so large yet during the outburst and at the beginning of quiescence, this would explain why we detected only the $f_{2}$ frequency.

In two other IP novae, V4743 Sgr and V407 Lup, the supersoft X-ray flux of the luminous central source before the end of nuclear burning decreased at almost constant temperature (Zemko et al. 2016; Aydi et al. 2018). This has been interpreted as an indication of a hot and luminous shrinking region on the WD, most likely consisting of the polar caps 

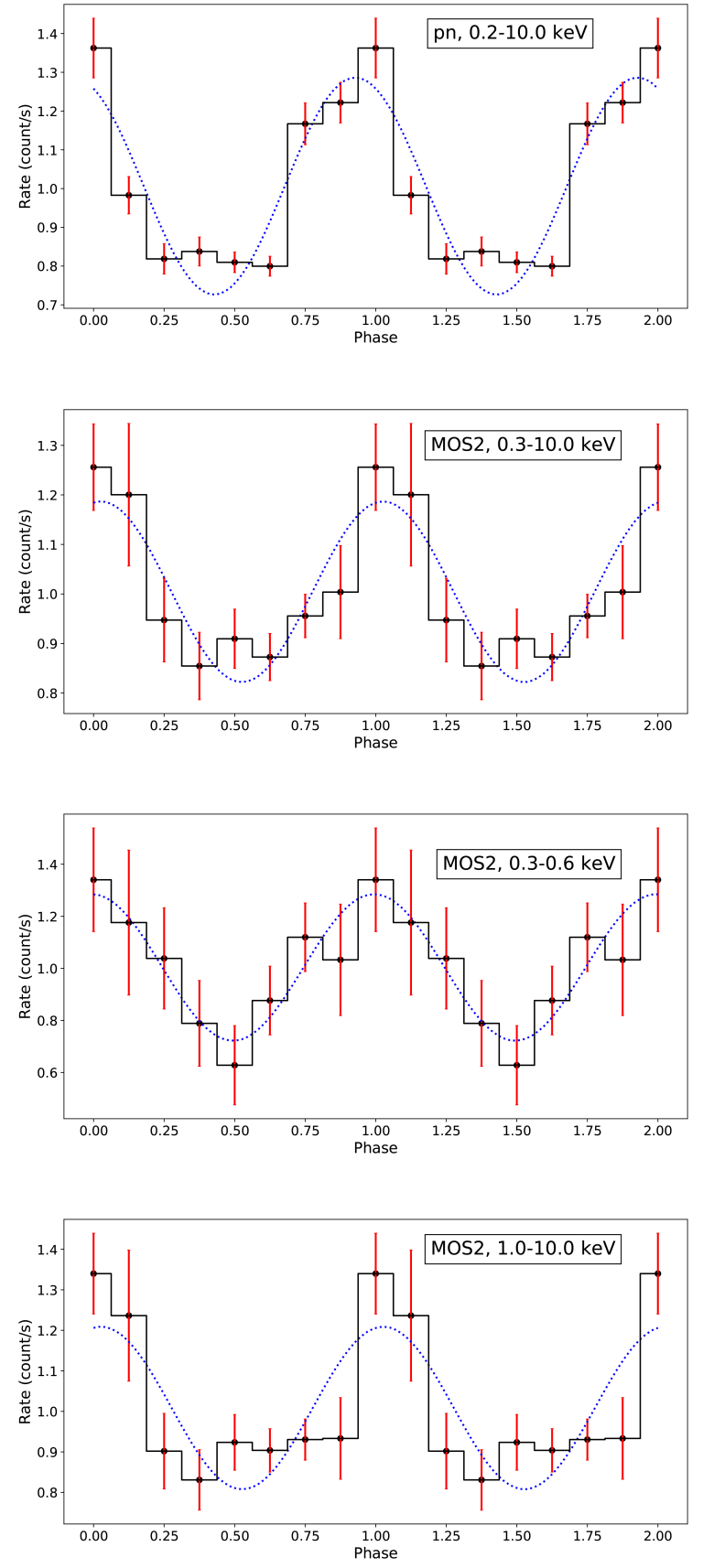

Figure 11. Light curves of EY Cyg folded with the orbital period, for the pn and for the MOS2. For the MOS2 we also present the very soft $(0.3-0.6 \mathrm{keV})$ and the hard $(1-10 \mathrm{keV})$ ranges. The count rate has been normalized to the average of each light curve, and the blue dots show a sine wave fit to the folded light curve, which assists to visualize the periodicity.

on which fresh matter is accreted, either keeping the high $\mathrm{T}_{\text {eff }}$ for longer, or fueling continued nuclear burning that remains confined to the deeper layers and does not expand in the rest of the surface. In V2491 Cyg we still detected large supersoft flux: possibly, this effect of polar accretion was still continuing.

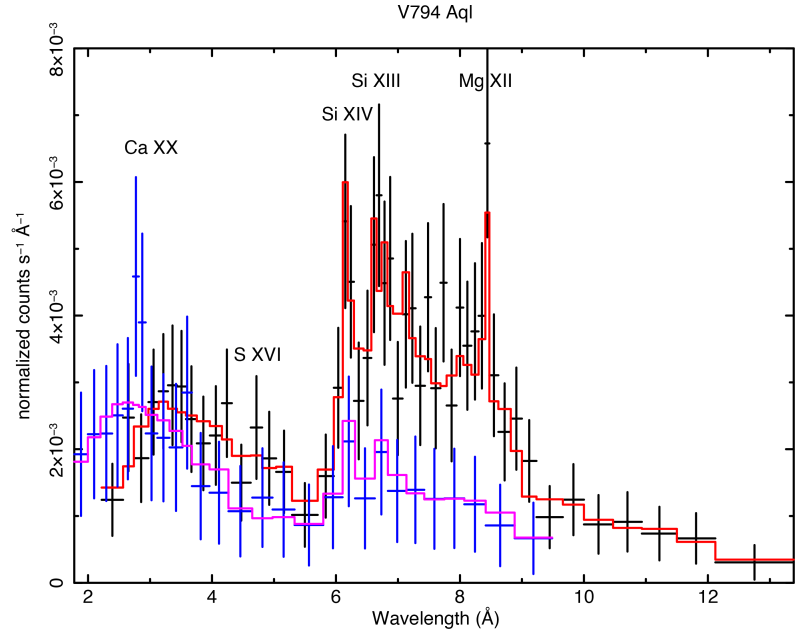

Figure 12. The HEG (in blue) and the MEG (in black) spectra of V794 Aql and the fit with Model 1. (in red for the MEG and pink for the HEG).

\subsection{X-ray luminosity probing accretion rate}

Despite several early findings that the X-ray flux of CVs may be too low to be due to energy dissipated in the accretion process (e.g. Mauche 1998; Orio et al. 2001, and references therein), the X-ray luminosity of V2491 Cyg is consistent with high accretion rate predicted for a nova of low amplitude and with similarities to RNe (see calculations by Yaron et al. 2005). KT Eri is at much lower X-ray luminosity, and the derived $\dot{m}$ is not consistent with the models' predictions for a nova with an optical amplitude of only 9 mag (Yaron et al. 2005). All the outburst models, in fact, generally indicate that the amplitude is larger if the nova accretes at low $\dot{m}$. This can be reconciled with what we observe only of this relatively small amplitude is caused by an optically luminous, evolved secondary.

We find that the X-ray luminosity of the dwarf nova and ex-CN candidate, EY Cyg, is consistent with the low $\dot{m}$ expected for dwarf novae and also, most likely, for "hibernating" novae. Recently Nabizadeh \& Balman (2020), shortly after our presentation at the 2020 AAS winter meeting (Sun et al. 2020), also examined our EY Cyg data, available in the HEASARC archive. These authors favor a multi-temperature plasma emission model (CEVMKL), analogous to VMFLOW if the power of the emission measure distribution is consistent with radiative cooling. Their fit indicates hydrogen column density $N_{H}=0.04_{-0.01}^{+0.02} \times 10^{22} \mathrm{~cm}^{-2}$, which is consistent with our result in Table 5 , but their result on the unabsorbed $\mathrm{X}$-ray flux is $19 \pm 1 \times 10^{-13} \mathrm{erg} \mathrm{cm}^{-2} \mathrm{~s}^{1}$ in the $0.1-50.0 \mathrm{keV}$ range. This flux is larger than what we obtain extrapolating the flux to $50 \mathrm{keV}$ and would indicate an even larger $\dot{m}$ than we obtained using Equation 1. However, Nabizadeh \& Balman (2020) derived $\dot{m}=6.0_{-0.3}^{+0.3} \times 10^{-12} \mathrm{M}_{\odot} \mathrm{yr}^{-1}$, which is only approximately half of what we estimated with the assumption that half of the gravitational energy is emitted as X-ray light. We disagree with these authors' conclusion that EY Cyg is underluminous in comparison with what is expected from the boundary layer of the disk and that invoking an advective flow is necessary to explain the data; we rather find that the $\dot{m}$ value is consistent with an approximate estimate 
obtained form the UV flux. There was no rigorous determination from the UV flux in Sion et al. (2004), in fact the given value can be considered only as upper limit.

Although we agree with Nabizadeh \& Balman (2020) in attributing the soft portion of the X-ray flux comes from the $\mathrm{K}$ star, the soft flux below $1 \mathrm{keV}$ is only 20 times lower than that of the harder portion, thus only borderline relevant in contaminating the result of the emission from accretion if it represents the X-ray flux of the secondary star. In the spectral range previously evaluated, $\sim \mathrm{K} 5-\mathrm{M} 0$ (late $\mathrm{K}$ to early M) (Smith et al. 1997), the X-ray luminosity is $10^{29}-10^{30} \mathrm{erg}$ $\mathrm{s}^{-1}$, but for K0 stars (Nabizadeh \& Balman 2020) quote a case in which the luminosity reached even $\simeq 10^{31} \mathrm{erg} \mathrm{s}^{-1}$. Thus the first APEC component in our Model 2 in the table, which has a temperature of $0.93 \mathrm{keV}$, may represent the (usually quite soft) contribution of the $\mathrm{K}$ star, but it yields only an absorbed flux of $5.5 \times 10^{-14} \mathrm{erg} \mathrm{cm}^{-2} \mathrm{~s}^{-1}$ and an unabsorbed flux of $6.5 \times 10^{-14} \mathrm{erg} \mathrm{cm}^{-2} \mathrm{~s}^{-1}$. This is negligible compared to the total X-ray flux, the bulk of which seems to be due to accretion.

\subsection{Are the VY Scl binaries nova-like accreting at high $\dot{m}$ during the whole high states period?}

One surprising result we obtained is the apparent lack of any clear correlation between the high and the low optical states of the nova-like VY Scl binary of our sample, V794 Aql, and its X-ray flux level. Moreover, the X-ray spectrum of V794 Aql is very hard: emission lines due to atomic transitions at lower energy than $1.5 \mathrm{keV}$ are absent. If the X-ray flux is indicative of accretion, a VY Scl binary like V794 Aql does not only have decreased $\dot{m}$ in the optically low states, but accretion perhaps is intermittent. This is a likely reason for which nuclear burning, with the manifestation of a luminous supersoft X-ray source, is not ignited (Zemko et al. 2014).

The plasma temperature of the hotter component in this nova-like exceeds $33 \mathrm{keV}$. It is one of the "hardest" CVs, and such a high temperature almost certainly indicates a massive WD, although we could not constrain the plasma temperature well. A more definite result may be obtained with the NuSTAR satellite, with the 3-79 keV bandpass of its X-ray telescope.

\section{CONCLUSIONS}

An important motivation to obtain a second exposure of both V2491 Cyg and KT Eri as they "settled" into quiescence was to monitor the supersoft X-rays, coming from a region that was apparently shrinking at a constant temperature. In V2491 Cyg, we found that this region is still luminous and hot. In KT Eri, we witness the hardening of the X-ray spectrum over a few years, but our analysis of the previous data obtained with Chandra indicates that the luminosity was already so low that it may have nothing to do with lasting heat on the polar caps due to long lasting nuclear burning underneath them. KT Eri does not show any signs of magnetic accretion, and is probably accreting only through a disk.

We derived high accretion rate, above $10^{-9} \mathrm{M}_{\odot} \mathrm{yr}^{-1}$ for V2491 Cyg, and much lower, about $2 \times 10^{-10} \mathrm{M}_{\odot} \mathrm{yr}^{-1}$ for KT Eri. We confirmed that V2491 Cyg has the characteristics of an IP, and we found an intriguing $\simeq 81$ minutes period in the X-ray light curve of EY Cyg, which leaves open the possibility that is another IP, although the high background of the exposure makes the result still uncertain.

An orbital modulation of the X-ray flux in EY Cyg, a low inclination system, may be due to the softest X-ray portion coming from the secondary. The $\dot{m}$ value, although low enough to be consistent with its dwarf nova outbursts, is not inconsistent with the UV luminosity attributed to the accretion disk.

We found that V794 Aql is a hard X-ray emitter, indicating a high mass WD, but we also discovered that $\dot{m}$ was quite lower than previously measured during optically high states. We concluded that $\dot{m}$ is probably very variable even during the high states. In the light of the recent nova outburst of a VY Scl nova-like system, these large fluctuations in $\dot{m}$ may also be a significant feature of nova systems, rather than a cyclic process of short periods of high $\dot{m}$ with "hibernation" in between.

\section{DATA AVAILABILITY}

The data analyzed in this article are all available in the HEASARC archive of NASA at the following URL: https:// heasarc.gsfc.nasa.gov/db-perl/W3Browse/w3browse.pl

\section{ACKNOWLEDGEMENTS}

M.O. is grateful to Koji Mukai for very useful discussions and help in the interpretation of the V794 Aql spectrum. M.O. also acknowledges NASA funding for the analysis of the XMM-Newton observations of EY Cyg, V2491 Cyg and KT Eri, and a Chandra award for V794 Aql. Support of an Italian INAF award dedicated to the search and study of targets that may be observed in the future with the Square Kilometer Array (SKA) and the Cerenkov Telescope Array (CTA) ("PRININAF 2017:Towards the SKA and CTA era: discovery, localization, and physics of transient objects") has helped putting this research together and making comparisons. A.D. was supported by the Slovak grant VEGA 1/0408/20. G.J,M.L. is a member of the CIC-CONICET (Argentina) and acknowledges support from grant ANPCYT-PICT 0901/2017. S.S.'s participation was supported by the Program of Development of M.V. Lomonosov Moscow State University "Leading Scientific Schools", project "Physics of Stars, Relativistic Objects and Galaxies", by the Slovak Academy of Sciences grant VEGA No. 2/0008/17, and by the Slovak Research and Development Agency under the contract No. APVV-15-0458,

\section{REFERENCES}

Aydi E., et al., 2018, MNRAS, 480, 572

Baklanov A., Pavlenko E., Berezina E., 2008, The Astronomer's Telegram, 1514, 1

Balman S., Orio M., Ogelman H., 1995, ApJ, 449, L47

Beardmore A. P., et al., 2010, The Astronomer's Telegram, 2423, 1

Bode M. F., et al., 2010, The Astronomer's Telegram, 2392, 1

Butters O. W., Norton A. J., Mukai K., Tomsick J. A., 2011, A\&A, 526, A77

Cash W., 1979, ApJ, 228, 939

Drake A. J., et al., 2009, The Astronomer's Telegram, 2331, 1 
Echevarría J., Michel R., Costero R., Zharikov S., 2007, A\&A, 462, 1069

Ferrario L., de Martino D., Gänsicke B. T., 2015, Space Sci. Rev., 191, 111

Fruscione A., et al., 2006, in Proc. SPIE. p. 62701V, doi: $10.1117 / 12.671760$

Godon P., Sion E. M., Barrett P., Szkody P., 2007, ApJ, 656, 1092

Helton L. A., Woodward C. E., Vanlandingham K., Schwarz G. J., 2008, Central Bureau Electronic Telegrams, 1379, 1

Hillman Y., Shara M. M., Prialnik D., Kovetz A., 2020, Nature Astronomy, 4, 886

Honeycutt R. K., Kafka S., Robertson J. W., 2014, AJ, 147, 10

Hounsell R., et al., 2010, The Astronomer's Telegram, 2558, 1

Ibarra A., et al., 2009, A\&A, 497, L5

Jurdana-Sepic R., Munari U., 2008, Information Bulletin on Variable Stars, 5839, 1

Jurdana-Šepić R., Ribeiro V. A. R. M., Darnley M. J., Munari U., Bode M. F., 2012, A\&A, 537, A34

Li K.-L., Kong A., Aydi E., Sokolovsky K., Chomiuk L., Kawash A., Strader J., 2020, The Astronomer's Telegram, 13868, 1

Mason E., et al., 2013, MNRAS, 436, 212

Mauche C. W., 1998, in Howell S., Kuulkers E., Woodward C., eds, Astronomical Society of the Pacific Conference Series Vol. 137, Wild Stars in the Old West. p. 113 (arXiv: astro-ph/9709101)

Morrison R., McCammon D., 1983, ApJ, 270, 119

Mukai K., Orio M., 2005, ApJ, 622, 602

Mukai K., Kinkhabwala A., Peterson J. R., Kahn S. M., Paerels F., 2003, ApJ, 586, L77

Munari U., Siviero A., Dallaporta S., Cherini G., Valisa P., Tomasella L., 2011, New Astron., 16, 209

Nabizadeh A., Balman S., 2020, arXiv e-prints, p. arXiv:2001.07486

Nakano S., Sumoto 2008, IAU Circ., 8934

Nelson T., Mukai K., Orio M., Luna G. J. M., Sokoloski J. L., 2011, ApJ, 737, 7

Ness J. U., Drake J. J., Starrfield S., Bode M., Page K., Beardmore A., Osborne J. P., Schwarz G., 2010, The Astronomer's Telegram, 2418, 1

Ness J. U., et al., 2011, ApJ, 733, 70

Ness J. U., et al., 2015, A\&A, 578, A39

Nixon C. J., Pringle J. E., 2020, A\&A, 636, A34

Orio M., 1993, A\&A, 274, L41

Orio M., Ogelman H., 1992, IAU Circ., 5680, 2

Orio M., Balman S., della Valle M., Gallagher J., Oegelman H., 1996, ApJ, 466, 410

Orio M., Covington J., Ögelman H., 2001, A\&A, 373, 542

Orio M., Mukai K., Bianchini A., de Martino D., Howell S., 2009, ApJ, 690, 1753

Orio M., et al., 2018, ApJ, 862, 164

Page K. L., et al., 2010, MNRAS, 401, 121

Pandel D., Córdova F. A., Mason K. O., Priedhorsky W. C., 2005, ApJ, 626, 396

Patterson J., Raymond J. C., 1985, ApJ, 292, 535

Ribeiro V. A. R. M., Darnley M. J., Bode M. F., Munari U., Harman D. J., Steele I. A., Meaburn J., 2011, MNRAS, 412, 1701

Scargle J. D., 1982, ApJ, 263, 835

Shara M. M., Livio M., Moffat A. F. J., Orio M., 1986, ApJ, 311, 163

Shaviv G., 2017, in The Golden Age of Cataclysmic Variables and Related Objects IV. p. 36

Shugarov S. Y., Chochol D., Volkov I. M., Zemko P. O., 2010, in Sterken C., Samus N., Szabados L., eds, Variable Stars, the Galactic halo and Galaxy Formation. pp 198-198

Sion E. M., Winter L., Urban J. A., Tovmassian G. H., Zharikov S., Gänsicke B. T., Orio M., 2004, AJ, 128, 1795

Smith R. C., Sarna M. J., CatalÃąn M. S., Jones D. H. P., 1997, Monthly Notices of the Royal Astronomical Society, 287, 271
Smith R. K., Brickhouse N. S., Liedahl D. A., Raymond J. C., 2001, ApJ, 556, L91

Starrfield S., Iliadis C., Timmes F. X., Hix W. R., Arnett W. D., Meakin C., Sparks W. M., 2012, Bulletin of the Astronomical Society of India, 40, 419

Sun B., Orio M., Dobrotka A., Luna G., Zemko P., 2020, in American Astronomical Society Meeting Abstracts. American Astronomical Society Meeting Abstracts. p. 452.03

Tomov T., Mikolajewski M., Brozek T., Ragan E., Swierczynski E., Wychudzki P., Galan C., 2008, The Astronomer's Telegram, 1485, 1

Tovmassian G., Orio M., Zharikov S., Echevarría J., Costero R., Michel R., 2002, in Hernanz M., José J., eds, American Institute of Physics Conference Series Vol. 637, Classical Nova Explosions. pp 72-76, doi:10.1063/1.1518181

Wilms J., Allen A., McCray R., 2000, ApJ, 542, 914

Wolf W. M., Bildsten L., Brooks J., Paxton B., 2013, ApJ, 777, 136

Yamaoka H., et al., 2009, IAU Circ., 9098, 1

Yaron O., Prialnik D., Shara M. M., Kovetz A., 2005, ApJ, 623, 398

Zemko P., Orio M., Mukai K., Shugarov S., 2014, MNRAS, 445, 869

Zemko P., Mukai K., Orio M., 2015, ApJ, 807, 61

Zemko P., Orio M., Mukai K., Bianchini A., Ciroi S., Cracco V., 2016, MNRAS, 460, 2744

Zemko P., et al., 2018, MNRAS, 480, 4489

This paper has been typeset from a $\mathrm{T}_{\mathrm{E}} \mathrm{X} / \mathrm{LAT} \mathrm{T} \mathrm{X}$ file prepared by the author. 